\title{
A point prevalence survey of antimicrobial agents and antibiotic susceptibility patterns of admitted patients in acute wards of a teaching hospital in Western India
}

\author{
Sanjay Jaiswal ${ }^{1}$, Sharan Shyam ${ }^{2 *}$ \\ ${ }^{\mathbf{1}}$ Associate Professor, ${ }^{2}$ Resident, Dept. of Pharmacology, Armed Forces Medical College, Pune, Maharashtra, India
}

*Corresponding Author: Sharan Shyam

Email: tsharanshyam@gmail.com

\begin{abstract}
Introduction: Antimicrobial stewardship programme (AMSP) is an important strategy to contain antimicrobial resistance (AMR) in the community.

Materials and Methods: A point prevalence survey was carried out in October 2018 in accordance with ECDC 2013 document. The survey was carried out on a single day in intensive care units, acute medical, acute surgical and family wards of a tertiary care hospital in western India.

Results: Less than $44 \%$ of admitted patients in acute wards were found to be prescribed with antimicrobial agents (AMA). The culture report of clinical specimen sent was positive for pathogen growth in $21 \%$ samples. Cephalosporins, penicillins and quinolones were the most commonly prescribed AMA on inpatients of the hospital. Of these, $70 \%$ were administered parenterally and $46 \%$ were given for more than one week. $70 \%$ of the empirical therapy was found to be effective. Pathogens resistant to many WHO WATCH group AMA and aztreonam, a RESERVE AMA have been isolated in the survey. Two of the isolated pathogens showed sensitivity to less than 3 antibiotics.

Conclusion: The present survey has revealed a high prevalence of infectious disease burden and antimicrobial usage in acute wards of our hospital.
\end{abstract}

Keywords: Point prevalence survey, Antimicrobial stewardship programme, Antimicrobial resistance, Antibiotic consumption.

\section{Introduction}

The emergence of antimicrobial resistance (AMR) presents a major threat to global public health. It is estimated that $50 \%$ or more of hospital antimicrobial use is inappropriate. ${ }^{1}$ Antibiotic resistance reduces the effectiveness and options for antibiotic treatment, leading to increased morbidity, mortality and health care expenditures.

A survey conducted by the Indian Council of Medical Research on antimicrobial stewardship programme (AMSP) in 20 healthcare institutions (HCI) in the year 2013, found good compliance with local guidelines on healthcare associated infections (HCAI) and hospital infection control measures. However, antimicrobial agents (AMA) usage data were analyzed by only $25 \%$ hospitals and AMA prescription audit and feedback by $30 \%$. Corporate/private hospitals performed better than government hospitals across all fields of AMSP. ${ }^{2}$

Two broad strategies to tackle the challenge of AMR are antimicrobial stewardship and hospital infection control. ${ }^{3}$ Multicenter point prevalence survey (PPS) is necessary to estimate the epidemiology of infections and antibiotic use especially in low and middle- income countries. It is of tremendous value to monitor restriction or rotation policies for AMA suggested on prevalent resistance patterns. ${ }^{1}$

Information regarding hospital antibiotic use in developing countries is still limited. The aim of the present study was to carry out a periodic prevalence survey on infectious diseases in a tertiary care hospital along with sensitivity pattern of pathogens isolated from clinical specimen.

\section{Materials and Methods}

A point prevalence survey was carried out in the first week of October 2018, in accordance with the European Centre for Disease Control technical document titled 'Point prevalence survey on healthcare-associated infections and antimicrobial use in European acute care hospitals'. ${ }^{4}$ The present descriptive study was approved by the Institutional Ethics Committee. The survey covered four acute wards of the hospital namely intensive care units (ICU), acute medical, acute surgical, and family wards. Each ward was covered on a single day. The bed state as on the day of the survey was recorded and the bed occupancy was calculated.

Patients admitted for day care procedures and those discharged from the wards on the day of survey, were not included. The data of patients admitted in these wards, was manually recorded using the case record forms from the patients' case sheets, investigation reports and nursing records. Patients put on antibiotics were followed up, and outcome of treatment was recorded.

The AMA not taken by the patient on the day of survey was not considered. Anti-virals, anti-fungals, antitubercular and anti-helminthic prescriptions were excluded from the study. The clinical samples of infectious diseases, sent to laboratory, were followed up for antibiotic sensitivity report. The case sheets were also studied for any change in the antibiotic regimen during the course of treatment of infections.

Antibiotic use was labeled as prophylactic, empirical and definitive. The day of start of empirical therapy was considered as 'day zero'. The $\mathrm{n}^{\text {th }}$ day from day zero was the point at which empirical therapy was changed to definitive therapy. The diagnosis of all the patients on antibiotics was also recorded. 


\section{Results \\ Hospital data (point survey of antibiotics in admitted hospital patients)}

The study was conducted in a teaching hospital of western India authorized a total of 1082 beds. Four wards of the tertiary care hospital namely ICU, acute medical, acute surgical and family wards, were selected for the present study.

Out of a total of 154 beds authorized to these four wards, 105 patients were found admitted during the survey $(68 \%$ bed occupancy). Of the 105 patients admitted, only 46 patients (less than 44\%) were found to be prescribed with antibiotics on the day of survey.

\section{Patient demography}

The demographic and clinical data of the patients on AMA is summarized in Table $1.65 \%$ of the admitted patients on antibiotics were below the age of 50 years. $70 \%$ of the inpatients on antibiotics were in the body weight range of 50 to $70 \mathrm{~kg}$.

\section{Infectious disease burden}

Table 2 gives the details of patients prescribed with antibiotics on the day of survey. 61 clinical samples for bacterial culture were sent, of which 13 were found to be positive for bacterial growth ( $21 \%$ positivity approximately). The most common antibiotic groups prescribed were cephalosporins $(22 \%)$, penicillins $(13 \%)$ and quinolones $(13 \%)$ as depicted in figure 1 . The maximum prevalence of antibiotic use was in the hospital ICU (79\%) and acute medical wards $(45 \%)$.

Out of 46 patients found to be prescribed with antibiotics, $15(33 \%)$ were on more than 2 antibiotics and 4 patients $(9 \%)$ were on more than 3 antibiotics. Of the total antibiotic prescriptions, $46 \%$ were prescribed for more than 1 week and $11 \%$ were prescribed for more than 2 weeks. $70 \%$ of the prescribed antibiotics were administered by parenteral route and the rest by oral route.

\section{Sensitivity results}

The treatment details of infectious diseases with positive culture report are mentioned in Table 3.

A total of 14 pathogens were isolated and summarized in Table 4 (one sample of pus showed growth of 2 pathogens). Organisms isolated, and their antibiotic sensitivity pattern has been highlighted in the above table.

In our study, pathogens which showed in-vitro resistance to the antibiotics tested were 12 in number, as mentioned in Table 5.2 of the isolated pathogens showed sensitivity to less than 3 antibiotics (Burkholderia capaciae and Escherichia coli). 8 out of the 12 pathogens were resistant to more than 5 antibiotics. 5 pathogens were isolated from pus culture, 4 from urine, 3 from blood and 2 from high vaginal swabs.

4 samples came positive for E.coli, 2 for P.mirabilis, 2 for E.faecium and 1 each for B.capaciae, P.aeruginosa, E.avium, coagulase negative Staph aureus.

$50 \%$ of the infectious patients were continued on the empirical therapy despite the sensitivity report to alternative antibiotics, since the patients showed signs of clinical improvement. In our study, $70 \%$ of the empirical antibiotic therapy started (including 2 antibiotics where only dosage form was changed) was found to be effective and did not require any change on obtaining the culture report. The preferred empirical antibiotics for pneumonia were ceftriaxone and levofloxacin.

30 infectious patients had negative culture reports. In these patients, the preferred antimicrobial agents for empirical therapy were ceftriaxone (10 patients), azithromycin (7 patients), metronidazole (6 patients), levofloxacin (7 patients) and meropenem (4 patients). Even in such patients, change in antibiotics was required in less than $7 \%$ of the admitted patients.

\section{Discussion}

There is a paucity of data describing hospital associated infections and antibiotic usage in developing countries. Reporting and sharing data on AMA consumption pattern is an essential element of surveillance and fight against AMR. The WHO Model List of Essential Medicines has categorized antibiotics into three groups: ACCESS, WATCH and RESERVE. WHO has cautioned the use of broad - spectrum antibiotics such as third generation cephalosporins, quinolones and carbapenems because of their high potential to cause selection of AMR and/or their side-effects. ${ }^{5}$ Such WATCH antibiotics consumption has varied from less than $20 \%$ in some countries to more than $50 \%$ in others. ${ }^{6}$

Hospital based infectious diseases surveillance programmes may focus on specific locations in the health care facility (e.g. intensive care unit, adult acute wards). Hence, the present survey has been carried out in areas expected to have the highest burden of infectious diseases in the hospital.

A WHO community-based pilot project revealed that 36$40 \%$ prescriptions in western India had antibiotics prescribed and the most commonly used AMA were extended spectrum penicillins, cotrimoxazole, tetracycline and fluoroquinolones. AMA with more than $50 \%$ isolates showing resistance was ampicillin, nalidixic acid and tetracycline. $^{7}$

The Global - PPS data for 2015 from 303 hospitals in 53 countries showed highest frequency of hospital associated infections in Latin America (11.9\%) and east and south Asia $(10.1 \%)$. At least $34.4 \%$ received one antimicrobial in their hospital stay. The top three antibiotics prescribed worldwide were penicillins with $\beta$ - lactamase inhibitors, third generation cephalosporins and fluoroquinolones. ${ }^{8}$ Unfortunately, India has one of the highest prevalence of antimicrobial resistance in the world. ${ }^{9}$ India was the largest consumer of antibiotics for human health in 2010, although the per capita consumption (10.7 units per capita) was lower than that seen in many developed nations (e.g. 22 units per capita in US).$^{10}$ An OPD based study from India highlighted extremely high AMA consumption across public sector, private retail sector and private clinics. ${ }^{11}$ Our snapshot study is in conformity to WHO report, stating that the level of consumption of thirdgeneration cephalosporins is still very high. ${ }^{6}$

In an African survey, a pathogen was isolated for $12.5 \%$ of HCAI, the most common being E.coli. Approximately $61 \%$ of all patients received at least one antimicrobial agent 
on the survey day. ${ }^{12}$ A PPS done in western Kenya reported $67.7 \%$ of admitted patients on antibiotics. The most common classes of antibiotics prescribed were cephalosporins $(55 \%)$ followed by broad spectrum penicillins $(41.8 \%) \cdot{ }^{13}$ In a survey on Iranian pediatric hospitals, $66.6 \%$ of admitted children received AMA of which therapeutic use was $60.6 \%$. Ceftriaxone was the most prescribed antimicrobial $(32.4 \%) .{ }^{14}$

In a hospital-based PPS on neonates in India, it was reported that $51.6 \%$ were prescribed with one or more AMA. Of these, $74.5 \%$ were prescribed AMA for an active infection. Amikacin followed by meropenem were the two most commonly prescribed AMA. ${ }^{15}$

A PPS on Chinese hospitals reported $56 \%$ prevalence of antibiotic use among admitted patients. The most commonly used AMA were $\beta$ - lactam AMA including cephalosporins $(40 \%)$ and penicillins (19\%) followed by fluoroquinolones $(14 \%) .{ }^{16}$ Acute care hospitals survey in Singapore showed $11.9 \%$ HCAI among admitted patients, of which $45.5 \%$ were culture positive. $51 \%$ of the patients were on systemic AMA of which $12.8 \%$ were prescribed for surgical prophylaxis. Amoxicillin/ clavulanate was the most frequently prescribed AMA (24.6\%). ${ }^{17}$

A pilot cross-sectional PPS at a Japanese University hospital showed that $10.1 \%$ of administered patients had HCAI and $33.6 \%$ were administered AMA. Carbapenems $(22.9 \%)$ were the most frequently administered category of AMA. ${ }^{18}$

A survey on medical and surgical wards of a teaching hospital in Italy showed $53.9 \%$ of patients receiving AMA. ${ }^{9}$ Again, the 2016 prevalence of HCAI in public hospitals of Italy was estimated to be $10.3 \%$ and $46 \%$ of patients received at least one AMA. Penicillin along with $\beta$ - lactamase inhibitor was the most widely used $(24.1 \%){ }^{20}$

In a survey of Slovak hospitals, $30.7 \%$ of inpatients were found receiving AMA. Fluoroquinolones (20.9\%) were the most frequently used AMA. ${ }^{21} 33.8 \%$ of the patients admitted in the surveyed wards of a Dutch university hospital were administered AMA. Of these, $22.2 \%$ were for therapeutic use of infectious diseases. ${ }^{22}$ A Norwegian study reported $8 \%$ use of AMA in hospitals for prophylaxis. ${ }^{23}$ Further, $40.9 \%$ of children hospitalized on the day of PPS were found to be prescribed AMA in a UK - based study. ${ }^{24}$

The present survey has revealed a high prevalence of infectious disease burden and antimicrobial usage in acute wards of our hospital. But the burden of infections is still very high even in developed nations and needs coordinated efforts to reduce the existing prevalence. While further research is necessary to understand the causes and costs of HCAI and antimicrobial usage, repeated PPS will be useful to measure the progress in controlling these concerns. The present study further emphasizes the need to implement the AMSP even more stringently in the hospital.

Treatment failure in antimicrobial therapy often results due to selective pressure of resistant microbes to multiply and ultimately replace the susceptible bacteria population with resistant pathogens. A need has therefore, been felt to tighten regulatory control over use of newer and high-end antibiotics in order to preserve these as reserve drugs for life threatening infections. ${ }^{25}$

Empirical therapy should be practiced only if there is scientific evidence to support such a protocol. An effective hospital infection control programme and universal precautions can reduce the empirical use of AMA. Further, it is essential that the antimicrobial spectrum of AMA be narrowed down at the earliest, based on clinical judgement and antibiotic susceptibility tests.

The World Health assembly has come up with the Global Action Plan to contain AMR (GAP-AMR) in May 2015. It has been realized that newer antibiotic classes are slow to develop and investments by the industry towards antimicrobials are waning. It is alarming to note that by 2014 , the proportion of isolates resistant to methicillin had risen to $47 \%$ in India whereas it has actually been decreasing in developed nations. Periodic surveillance and research and regular analysis of the use of AMA are therefore, important strategies to contain AMR. ${ }^{26}$

The national data of 2014 for AMR of Staphylococcus aureus stated $35.7 \%$ resistance to cefoxitin and $45.7 \%$ resistance to cotrimoxazole whereas strains encountered in our survey were still sensitive to these drugs while being resistant to ciprofloxacin. For Salmonella spp. whereas the national data suggested almost $70 \%$ resistance to ciprofloxacin, the strain cultured in our survey on inpatients were sensitive to many drugs of even WHO ACCESS group. The strains of Pseudomonas cultured in our survey have been found to be sensitive to many ACCESS group medicines such as beta-lactams, gentamicin and ciprofloxacin when the national data has been around $40 \%$ being resistant. ${ }^{1}$ Amongst Enterobacteriaceae, strains cultured in the survey have remained resistant to beta-lactams, $3^{\text {rd }}$ generation cephalosporins but sensitive to amikacin and carbapenems. Strains of E. Coli sensitive to only cotrimoxazole or chloramphenicol is of concern as many WATCH group antibiotics are also found to be resistant. Pathogens resistant to aztreonam, a RESERVE antibiotic, is a significant and alarming finding in our survey.

In 2015, the Food Safety and Standards Authority of India issued a directive to limit the use of antibiotics as growth promoters in animal feed. The National AMR Containment Policy of India has acknowledged the need for a separate $\mathrm{H} 1$ schedule under the Drugs and Cosmetics Rules, to regulate the sales of AMA. It has been able to implement color-coded tagging antibiotics, and newer AMA (carbapenems, tigecycline, daptomycin, etc.) to eliminate their use outside tertiary care settings. ${ }^{27}$ A Red Line Campaign was launched in 2016 in India wherein all antibiotics are marketed with a red line on the package. The campaign aims to raise awareness against self-medication and use of AMA without a valid prescription with its potential harms. The National Treatment Guidelines for Antimicrobial Use in Infectious Diseases were released in 2016 to ensure rational use of AMA. ${ }^{3}$ 
Table 1: Demographic data of patients on antibiotics on day of survey

\begin{tabular}{|l|c|}
\hline Age & Number of patients \\
\hline $18-30$ & 14 \\
\hline $31-50$ & 16 \\
\hline $51-60$ & 5 \\
\hline$>60$ & 11 \\
\hline Sex & Number of patients \\
\hline Male & 27 \\
\hline Female & 19 \\
\hline Weight $(\mathbf{k g})$ & Number of patients \\
\hline$<50$ & 6 \\
\hline $50-70$ & 32 \\
\hline$>70$ & 8 \\
\hline
\end{tabular}

Table 2: Survey of inpatients of acute wards for antibiotic usage and culture report

\begin{tabular}{|c|c|c|c|c|}
\hline $\begin{array}{c}\text { Total number of } \\
\text { patients surveyed }\end{array}$ & $\begin{array}{c}\text { Number of patients } \\
\text { on empirical } \\
\text { antibiotics }\end{array}$ & $\begin{array}{c}\text { Total number } \\
\text { of infectious } \\
\text { patients }\end{array}$ & $\begin{array}{c}\text { Total number of } \\
\text { clinical specimen } \\
\text { sent for culture }\end{array}$ & $\begin{array}{c}\text { Total number of specimen } \\
\text { with positive culture report }\end{array}$ \\
\hline 105 & 6 & 40 & 61 & 13 \\
\hline
\end{tabular}

Table 3: Treatment analysis of infectious diseases with positive culture report

\begin{tabular}{|c|c|c|}
\hline \multirow{2}{*}{ Diagnosis } & Empirical therapy (day zero) & $\begin{array}{c}\text { Definitive therapy } \\
\text { (nth day when drug changed) }\end{array}$ \\
\cline { 2 - 4 } GI & Antibiotic name & $\begin{array}{c}\text { Antibiotic name } \\
\text { Imipenem }\end{array}$ \\
\hline OBGY & Faropenem (14)
\end{tabular}

GI- Gastrointestinal infections, ENT- infections of ear, nose, throat, larynx and mouth, FN- febrile neutropenia, PNEUpneumonia, OBGY- obstetric or gynecological infections, SST-O-cellulitis, wound or deep soft tissue not involving bone, not related to surgery, SST-SSI- Surgical site infection involving skin or soft tissue but not bone 
Table 4: Antibiotic susceptibility pattern of pathogens isolated from inpatients of acute ward

\begin{tabular}{|c|c|c|}
\hline Pathogen isolated (number of isolates) & AMA Sensitive (\% of isolates sensitive) & AMA Intermediate \\
\hline Burkholderia capaciae (1) & Meropenem & \\
\hline $\begin{array}{l}\text { Coagulase Negative } \\
\text { Streptococci (1) }\end{array}$ & $\begin{array}{l}\text { Cotrimoxazole (100\%), Linezolid (100\%), } \\
\text { Doxycycline (100\%), Teicoplanin }(100 \%), \\
\text { Amikacin (100\%), Tigecycline (100\%), Cefoxitin } \\
(100 \%), \text { Piperacillin }(100 \%)\end{array}$ & \\
\hline Enterococcus avium (1) & $\begin{array}{l}\text { Ciprofloxacin, Levofloxacin, Teicoplanin, } \\
\text { Vancomycin, Linezolid, Nitrofurantoin }\end{array}$ & Erythromycin (100\%) \\
\hline Enterococcus faecium (2) & $\begin{array}{l}\text { Tigecycline }(100 \%), \text { Vancomycin }(100 \%), \\
\text { Linezolid (100\%), Levofloxacin }(100 \%), \\
\text { Teicoplanin }(100 \%) \text {, Ciprofloxacin }(50 \%)\end{array}$ & \\
\hline Escherichia coli (4) & $\begin{array}{l}\text { Ciprofloxacin (50\%), Nitrofurantoin (75\%), } \\
\text { Amikacin (75\%), Cefotaxime (25\%), Ceftriaxone } \\
(25 \%) \text {, Levofloxacin (25\%), Ampicillin (25\%), } \\
\text { Ampicillin+Sulbactam (25\%),Amoxicillin (25\%), } \\
\text { Imipenem (50\%), Ertapenem (50\%), Meropenem } \\
(50 \%) \text {, Aztreonam (25\%), Cefepime (25\%), } \\
\text { Fosfomycin (25\%), Cefazolin (25\%), } \\
\text { Cotrimoxazole (50\%), Piperacillin+Tazobactam } \\
(25 \%) \text {, Norfloxacin (25\%), Chloramphenicol } \\
(25 \%)\end{array}$ & $\begin{array}{l}\text { Piperacillin+Tazobacta } \\
\text { m (25\%), Amoxicillin } \\
(25 \%) \text {, Teicoplanin } \\
(25 \%)\end{array}$ \\
\hline Proteus mirabilis (2) & $\begin{array}{l}\text { Imipenem (100\%), Meropenem (50\%), Ertapenem } \\
(50 \%) \text {, Amikacin (100\%), Aztreonam (50\%), } \\
\text { Linezolid (50\%), Ceftazidime (50\%), Ceftriaxone } \\
\text { (50\%), Cefotaxime (50\%), } \\
\text { Piperacillin+Tazobactam (50\%). }\end{array}$ & \\
\hline Pseudomonas aeruginosa (2) & $\begin{array}{l}\text { Ciprofloxacin (100\%), Tobramycin (50\%), } \\
\text { Amikacin (100\%), Imipenem (100\%), Meropenem } \\
(100 \%) \text {, Piperacillin+Tazobactam(100\%), Colistin } \\
(50 \%) \text {, Gentamicin }(50 \%)\end{array}$ & \\
\hline Salmonella typhi (1) & $\begin{array}{l}\text { Teicoplanin (100\%), Levofloxacin (100\%), } \\
\text { Ciprofloxacin (100\%), Meropenem }(100 \%), \\
\text { Cotrimoxazole (100\%), Amoxicillin (100\%), } \\
\text { Piperacillin (100\%), Tobramycin (100\%), } \\
\text { Ampicillin+Sulbactam (100\%), Ampicillin } \\
(100 \%), \text { Aztreonam (100\%), } \\
\text { Piperacillin+Tazobactam (100\%), Cefotaxime } \\
\text { (100\%), Ceftazidime (100\%), Ertapenem (100\%), } \\
\text { Imipenem (100\%), Cefepime (100\%) }\end{array}$ & \\
\hline
\end{tabular}

Table 5: Pathogens with list of antibiotics resistant (number of isolates positive in brackets)

\begin{tabular}{|l|c|c|c|c|c|c|}
\hline Burkholderia cepacia (1) & $\begin{array}{c}\text { Coagulase } \\
\text { negative } \\
\text { Streptococci (1) }\end{array}$ & $\begin{array}{c}\text { Enterococc } \\
\text { us avium } \\
\text { (1) }\end{array}$ & $\begin{array}{c}\text { Enterococcus } \\
\text { faecium (2) }\end{array}$ & $\begin{array}{c}\text { Escherichia } \\
\text { coli } \\
\text { (4) }\end{array}$ & $\begin{array}{c}\text { Proteus } \\
\text { mirabilis (2) }\end{array}$ & $\begin{array}{c}\text { Pseudomonas } \\
\text { aeruginosa (1) }\end{array}$ \\
\hline $\begin{array}{l}\text { Amoxicillin + clavulanic } \\
\text { acid }\end{array}$ & Ciprofloxacin & Gentamicin & Erythromycin & Amoxicillin & Ampicillin & Aztreonam \\
\hline Aztreonam & Levofloxacin & Meropenem & Gentamicin & Ampicillin & $\begin{array}{c}\text { Amoxicillin+ } \\
\text { Clavulanic acid }\end{array}$ & Cefepime \\
\hline Cefepime & Teicoplanin & $\begin{array}{l}\text { Piperacillin+ } \\
\text { Tazobactam }\end{array}$ & Norfloxacin & Aztreonam & Aztreonam & Ceftazidime \\
\hline Cefotaxime & & Teicoplanin & Tetracycline & Cefotaxime & Ciprofloxacin & Doxycycline \\
\hline Ceftriaxone & & & Ceftazidime & Cotrimoxazole & Imipenem \\
\hline Cotrimoxazole & & & & Ceftriaxone & Doxycycline & Levofloxacin \\
\hline Gentamicin & & & & Ciprofloxacin & Levofloxacin & \\
\hline Imipenem & & & & Doxycycline & Tetracycline & \\
\hline Piperacillin+Tazobactam & & & & Imipenem & & \\
\hline & & & & Meropenem & & \\
\hline
\end{tabular}




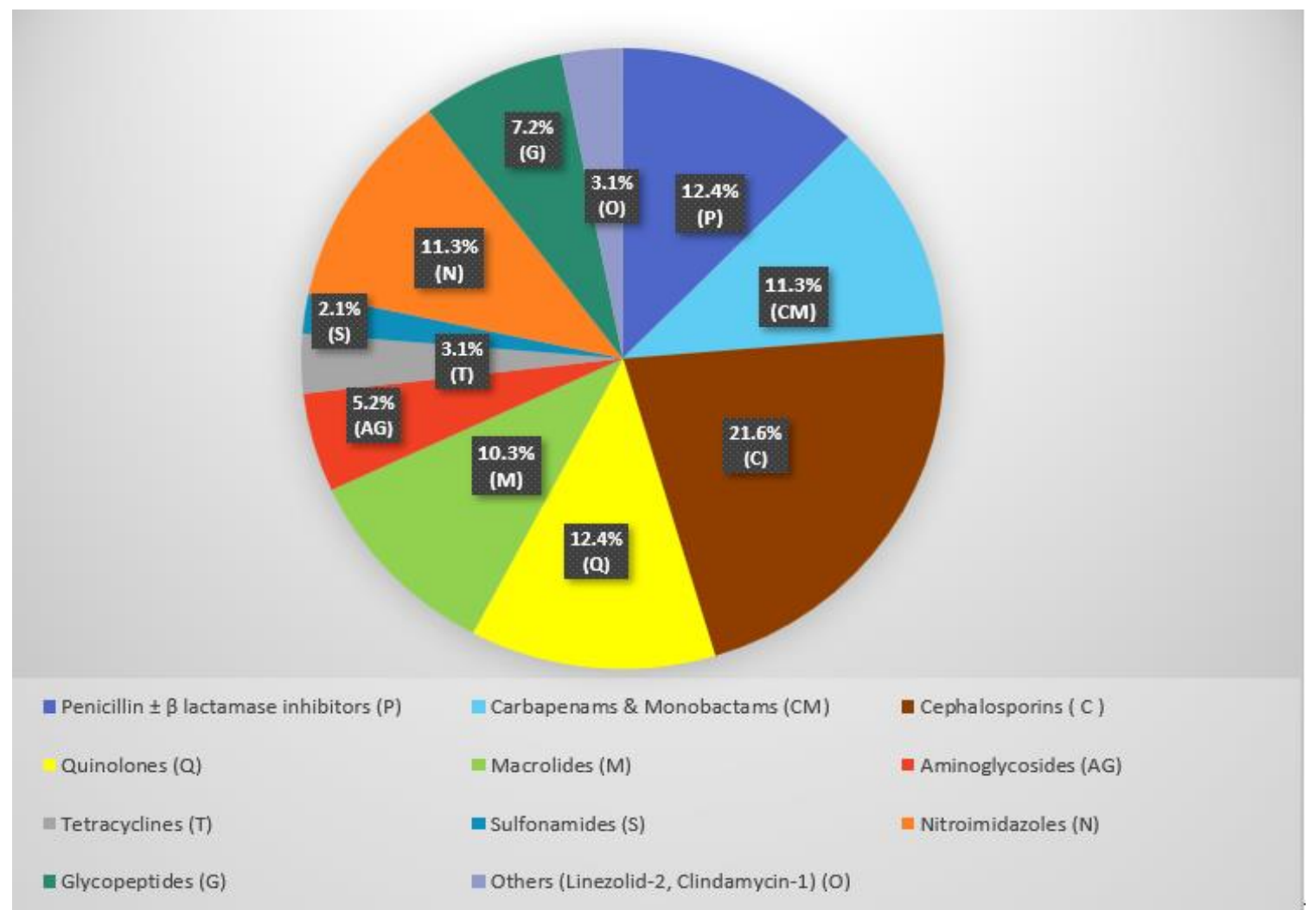

Note- Percentages in the above figure represent antibiotic consumption of the prescriptions surveyed

Fig. 1: Antibiotic consumption pattern of inpatients on the day of survey

\section{Conclusion}

The way forward then, is to have an effective AMSP with a multidisciplinary clinical team in place. The recommended members of this core team are an infectious disease physician, clinical pharmacist/ pharmacologist, clinical microbiologist, hospital epidemiologist and infection control nurse. Accreditation of healthcare institutions, prescription audits, user - friendly software on pathogen sensitivity, drug consumption patterns, infectious disease data record keeping with periodic analysis, continuous research and education are essential components at present to tackle HCAI. Finally, a sincere effort by all is the need of the hour to reduce or effectively treat infections in the community. In that context, this paper is a way forward to report and share data on antimicrobial usage pattern through a point prevalence survey in our hospital.

\section{Abbreviations}

AMA: Antimicrobial agents, AMSP: Antimicrobial stewardship programme

AMR: Antimicrobial resistance, HCAI: Healthcare associated infections

HCI: Healthcare institutions, PPS: Point prevalence survey WHO: World Health Organisation

\section{Strengths of the study}

The patients were followed up till discharged or transferred out from the ward. This has given the true duration of treatment, unlike other PPS where the duration of treatment is calculated only till the day of the survey and hence underestimated.

\section{Limitations of the study}

The study included only the acute wards of the hospital and therefore the antibiotic consumption data does not represent the entire hospital but the acute wards. The study was carried out in a single hospital and therefore may not reflect the overall antibiotic use in the region.

\section{Funding}

This research did not receive any specific grant from funding agencies in the public, commercial, or not-for-profit sectors.

\section{Author contributions}

SS acquired the data and performed the analysis. SJ conceptualized and designed the study and drafted the manuscript. Both authors reviewed, revised and approved the manuscript.

\section{Conflicts of interest}

The authors declare no conflict of interest.

\section{References}

1. ICMR. Department of Health Research New Delhi, India 2017. Treatment Guidelines for Antimicrobial Use in Common Syndromes.

2. https://www.icmr.nic.in/sites/default/files/guidelines/treatment _guidelines_for_antimicrobial.pdf [accessed 15 May 2019]. 
3. Walia K, Ohri VC, Mathai D. Antimicrobial Stewardship Programme of ICMR. Antimicrobial stewardship programme (AMSP) practices in India. Indian J Med Res 2015;142:130-8.

4. NCDC. Directorate General of Health Services, Ministry of Health \& Family Welfare, Government of India. National Treatment guidelines for Antimicrobial Use in Infectious Diseases. Version 1.0 (2016)

https:apps.who.int/medicinedocs/documents/ s23118en/s23118.pdf [accessed 15 May 2019]

5. European Centre for Disease Prevention and Control. Point prevalence survey of healthcare-associated infections and antimicrobial use in European acute care hospitals Stockholm: ECDC; 2013

6. WHO Model List of Essential Medicines. 20th List (March 2017). https://www.who.int

/medicines/publications/essentialmedicines/en/ [accessed 15 May 2019]

7. WHO report on surveillance of antibiotic consumption: 20162018 early implementation. Geneva: World Health Organization; 2018.https://who.int/medicines /areas/rational_use/who-amr-amc-report-20191109.pdf [accessed 15 May 2019]

8. World Health Organization. Community - based surveillance of antimicrobial use and resistance in resource-constrained settings report on five pilot projects. Geneva: World Health Organization; 2009.

https://www.who.int/medicines/publications/ community_based_may09.pdf [accessed 15 May 2019]

9. Versporten A, Zarb P, Caniaux I, Gros MF, Drapier N, Miller $\mathrm{M}$, et al. Antimicrobial consumption and resistance in adult hospital inpatients in 53 countries: results of an internetbased global point prevalencesurvey. Lancet Glob Health 2018;6:e619-e29. doi: 10.1016/S2214-109X(18)30186-4.

10. Gandra S, Singh SK, Jinka DR, Kanithi R, Chikkappa AK, Sharma A, et al. Point Prevalence Surveys of Antimicrobial Use among Hospitalized Children in Six Hospitals in India in 2016. Antibiotics 2017;6:19. doi:10.3390/antibiotics6030019.

11. Van Boeckel TP, Gandra S, Ashok A, Caudron Q, Grenfell BT, Levin SA, et al. Global antibiotic consumption 2000 to 2010: An analysis of national pharmaceutical sales data. Lancet Infect Dis 2014;14:742-50.

12. Kotwani A, Holloway K. Trends in antibiotic use among outpatients in New Delhi, India. BMC Infect Dis 2011;11(1):99.

13. Labi AK, Obeng-Nkrumah N, Owusu E, Bjerrum S, BediakoBowan A, Sunkwa-Mills G, et al. Multi-centre pointprevalence survey of hospital-acquired infections in Ghana. $J$ Hosp Infect 2019;101:60-8. doi:10.1016/j.jhin.2018.04.019.

14. Okoth C, Opanga S, Okalebo F, Oluka M, Baker Kurdi A, Godman B. Point prevalence survey of antibiotic use and resistance at a referral hospital in Kenya: findings and implications. Hosp Pract 2018;46:128-36. doi:10.1080/21548331.2018.1464872.

15. Fahimzad A, Eydian Z, Karimi A, Shiva F, Sayyahfar S, Kahbazi M, et al. Surveillance of Antibiotic Consumption Point Prevalence Survey 2014: Antimicrobial Prescribing in Pediatrics Wards of 16 Iranian Hospitals. Arch Iran Med 2016;19:204-9. doi: 0161903/AIM.009.

16. Gandra S, Alvarez-Uria G, Murki S, Singh SK, Kanithi $\mathrm{R}$, Jinka DR, et al. Point prevalence surveys of antimicrobial use among eight neonatal intensive care units in India: 2016. Int J Infect Dis 2018;71:20-4. doi:10.1016/j.ijid.2018.03.017.

17. Xie DS, Xiang LL, Li R, Hu Q, Luo QQ, Xiong W. A multicenter point-prevalence survey of antibiotic use in 13 Chinese hospitals. J Infect Public Health 2015;8:55-61. doi:10.1016/j.jiph.2014.07.001.
18. Cai Y, Venkatachalam I, Tee NW, Tan TY, Kurup A, Wong SY, et al. Prevalence of Healthcare-Associated Infections and Antimicrobial Use Among Adult Inpatients in Singapore Acute-Care Hospitals: Results From the First National Point Prevalence Survey. Clin Infect Dis 2017;64:S61-S7. doi: 10.1093/cid/cix103.

19. Morioka H, Hirabayashi A, Iguchi M, Tomita Y, Kato D, Sato $\mathrm{N}$, et al. The first point prevalence survey of health careassociated infection and antimicrobial use in a Japanese university hospital: A pilot study. Am J Infect Control 2016;44:e119-23. doi: 10.1016/j.ajic.2016.03.045.

20. Marani A, Napoli C, Berdini S, Montesano M, Ferretti F, Di Ninno F, et al. Point prevalence surveys on healthcare acquired infections in medical and surgical wards of a teaching hospital in Rome. Ann Ig 2016;28:274-81. doi: 10.7416/ai.2016.2106.

21. Sticchi C, Alberti M, Artioli S, Assensi M, Baldelli I, Battistini A, et al. Regional point prevalence study of healthcareassociated infections and antimicrobial use in acute care hospitals in Liguria, Italy. J Hosp Infect 2018;99:8-16. doi: 10.1016/j.jhin.2017.12.008.

22. Štefkovičová M, Litvová S, Meluš V, Krištúfková Z, Bražinová A. Point prevalence study of antimicrobial usage in acute care hospitals in the Slovak Republic. J Hosp Infect 2016;93:403-9. doi: 10.1016/j.jhin.2016.02.017.

23. Akhloufi H, Streefkerk RH, Melles DC, de Steenwinkel JE, Schurink CA, Verkooijen RP, et al. Point prevalence of appropriate antimicrobial therapy in a Dutch university hospital. Eur J Clin Microbiol Infect Dis 2015;34:1631-7. doi: 10.1007/s10096-015-2398-6.

24. Holen, Alberg T, Blix HS, Smith I, Neteland MI, Eriksen HM. Broad-spectrum antibiotics in Norwegian hospitals. Tidsskr Nor Laegeforen 2017;137:362-366. doi: 10.4045/tidsskr.16.0622. eCollection 2017 Mar. [accessed 15 May 2019]

25. Gharbi M, Doerholt K, Vergnano S, Bielicki JA, Paulus $\mathrm{S}$, Menson E, et al. Using a simple point-prevalence survey to define appropriate antibiotic prescribing in hospitalised children across the UK. BMJ Open 2016;6:e012675. doi: 10.1136/bmjopen-2016-012675.

26. World Health Organization. Antimicrobial resistance and its containment in India. Ministry of Health and Family Welfare, Government of India. New Delhi;2016 NCDC. National policy for containment of antimicrobial resistance. India. 2011. https://www.ncdc.gov.in/ab_policy.pdf [accessed 15 May 2019]

27. WHO. Antibiotic prescribing and resistance: Views from lowand middle income prescribing and dispensing professionals. https.//www.who.int/antimicrobial-resistance/LSHTMAntibiotic-Prescribing-LMIC- Prescribing-and-Dispensing2017.pdf [accessed 15 May 2019]

28. NCDC. National policy for containment of antimicrobial resistance. India. 2011. https://www.ncdc.gov.in/ab_policy.pdf [accessed 15 May 2019]

How to cite this article: Jaiswal S, Shyam S. A point prevalence survey of antimicrobial agents and antibiotic susceptibility patterns of admitted patients in acute wards of a teaching hospital in Western India. Indian J Pharm Pharmacol 2019;6(3):86-92. 